\title{
Quality Assurance: The Model of Quality Evaluation of Sport Exercises Achievement
}

\author{
Suharjo ${ }^{1}$ Imran Akhmad ${ }^{2}$, Rahma Dewi ${ }^{3}$ \\ ${ }^{1}$ Faculty of Sport Science, Medan State University Indonesia \\ ${ }^{2}$ Faculty of Sport Science, Medan State University Indonesia \\ ${ }^{3}$ Faculty of Sport Science, Medan State University Indonesia \\ ${ }^{1}$ Corresponding author: suharjo8564@gmail.com
}

\begin{abstract}
This research is aimed to establish the model of quality evaluation of the achievement sport exercises. This study generated some products such as: (1) document of quality, which includes; a) exercises quality standard, b) procedures manual, c) works instruction (2) developing instruments of quality evaluation of the achievement sport exercises. This research used research and development approach and was divided in three parts, such as (1) needs analysis, (2) develop the instruments, and (3) implementation of the outcome and evaluation to discover the quality of exercises performed by clubs or achievement sports association in order to improve the quality of exercises continuously. This research used research and development approach. This study concluded that the model of quality of exercises can possibly be used to strengthen the quality progressively. Evaluation of exercises quality is undertaken based on the standard of sports quality compared to the real condition.
\end{abstract}

Keyword: Evaluation, Quality, Exercise, Sport

\section{Introduction}

Sport accomplishment in North Sumatera is inconsistently moving and tend to decrease slightly. It could be clearly seen from the result of the Multi-Event Championship as well as National Sport Week. Furthermore, North Sumatera has not been standing at Top-Three position in the last six National Sport Week period, and even worse tend to drop moderately.

As a measure to support many achievements, Indonesian National Sport Committee of North Sumatera has launched several programs such as Intensive Guidance Program, aimed to increase the level in National Sport Week which held in Riau in 2012 and West Java. The aim that mentioned above is to penetrate the top-five position by winning twenty gold medals and also by applying continuous development program. That program unfortunately has failed according to the result of North Sumatera achievement which only stood at rank 9 . However, this particular program gained financial support from Provincial Spending Budget in order to obtain the Top-Three position in National Sport Week 2020. In sequence to maximize PPI program, then there are several things need to be done like measuring and revitalization, yet the most fundamental issue is the quality issue.

In the other hand, the progress of sport development based on the study held by Division of Economics Faculty, University of Indonesia in 2011 concluded: 59\% increase, $24 \%$ even, $17 \%$ decrease. Nonetheless, according to satisfaction index of Indonesian satisfaction towards sports in Indonesia could be seen with certain variables, Accomplishment (71.85\%), Event (79.24\%), Impression (77.13\%), facilities $(59.36 \%)$, establishment $(63.86 \%)$, welfare $(58.73 \%)$ and regeneration $(67.09 \%)$. Researchers believe that the service quality theory is based on the literature of customer satisfaction and product quality (Brady \& Cronin, 2001)

Azmi and Sunarno (2015) discovers the indicators of exercises quality exhibited lowest point compared to others indicators which reached $36 \%$. The most fundamental problem is most of the coaches do not have an obvious program training which is one of the most important thing for coaches.

Prevus in Fernandes (1986), says evaluation to figure the suitabily of degree between the decided standards in the real performance form. While the gaps that can be evaluated are: (1) The gap between planning and implementing the program, (2) The gap between suspected or predicted to be acquired and truly realized, (3) The gap between the status capabilities with standard capabilities defined, (4) Gaps purposes, (5) the gap of the parts of the program can be changed, and (6) 
gaps in the system inconsistent.

Implementation of quality control is done by using a management PDCA model (Plan, Do, Check, Action), which results the continuous quality development (improvement countinous) Abdullah (2015).

\section{Research Method \\ Procedure of Development}

This research used research and development approach (Borg and Gall: 1989) which grouped in 3 stages, such as: (1) PreDevelopment stage, (2) Development Stage, and (3) Application Stage.

\section{Results And Discussions}

\section{A. The Evaluation Model of Quality in Achievement Sport Exercises.}

A conceptual model of evaluation of exercises quality will be elaborated down below:

\section{Document Quality}

The system of quality assurance is done according to documents/scripts/quality/books, which consisted of:

a. The scripts/documents/books of quality policy, is a written document consisted of an explanation about how organizations understanding, planning and implementing quality assurance in implementation of quality assurance so that the quality culture will be built in that particular organization.

b. The manual Scripts/documents/books of quality procedure, consisted of practical guide about how SPM could be done, evaluated, and improved progressively by the responsible party to undertake in all of the direction.

c. Scripts/documents/books of quality standard, is a written document consisted of several criterias, sizes, specification of all the sport coaching events, to realize the vision and mission so the quality itself could be assessed according to the provision so it could satisfy the person in charge both internally and externally.

d. Scripts/documents/books of form/instruments, is a written document which serves to note/record some things or informations or certain events as an unseparatable part of quality standard, quality manual or quality procedure.

\section{Internal Quality Assurance}

As a material control in internal level, is directed to good pratical implementation for all the sources in organizing quality improvement continously for all organization in indonesia. This particular thing is done with the purpose of maintaining and improving the quality of sports training students run by an organization internally to realize the vision and meet the demands of the public about the need for the establishment of the organization and continuous quality improvement. Generally, Planning, Implementation, controlling, and improving the quality standar of organization consistantly and continuously so that will be having atlets who can compete globally.

To ensure the quality of the internal level, it is necessary to establish the Internal Quality Assurance by the manager in every organization in charge of guarding, maintaining and ensuring good practice has been done throughout the organization. Orientation internal quality control is to internalize internal quality culture in the organization.

\section{External Quality Control}

Quality control (quality control) conducted by outsiders who aim to give recognition from outsiders, especially stakeholders as material to measure the achievement level of each organization in Indonesia. To control the quality is performed by independent institution created by policy makers that objectively done by using instruments according to the quality standard document.

Implementation of quality control is done by stakeholders usefully to determine the policy for the management of the organization in Indonesia. It means, The results of the assessment instrument as a material for stakeholders to determine the sustainability of organizations based in Indonesia through accreditation.

In order to guarantee the implementation and the quality control is done through evaluation technique with instruments developed with reference to the quality standards that have been set on quality documents. 


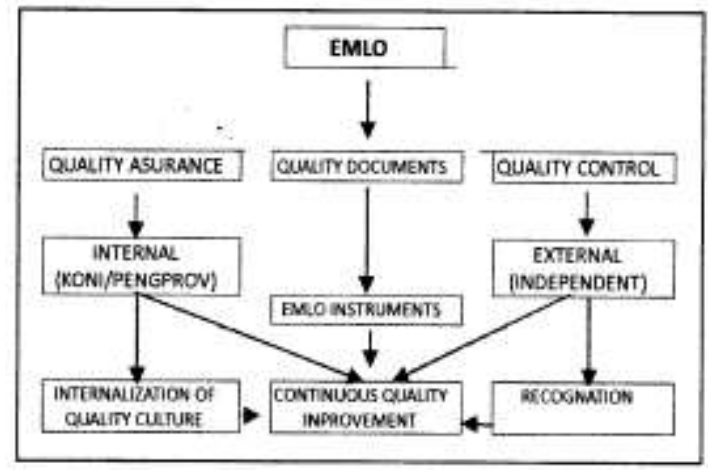

Figure 1. Model Evaluation of Sport Exercises Quality.

\section{B. The Result Of Evaluation \\ 1. Content Standard Training Program}

The evaluation results show the answers of items (1.1) clarity and completeness of policy documents regarding the establishment and development of training programs, $(50 \%)$ respondents chose items no (4). The answers of item test (1.2) the involvement of stakeholders (coaches, athletes, ex-athletes, and users) in order to arrange training programs, $(50 \%)$ respondents chose item no (5). The answers of item test (1.3) the clarity of documents and guidance in preparation and development training program and evaluation of training program evaluation, $(50 \%)$ respondents chose item test no. (5). The answers of item test (1.4) the clarity and complexity of training program's document along with science Improvement and the need of sports, (30\%) respondents chose item no (5). The result of tabulation can be seen below:

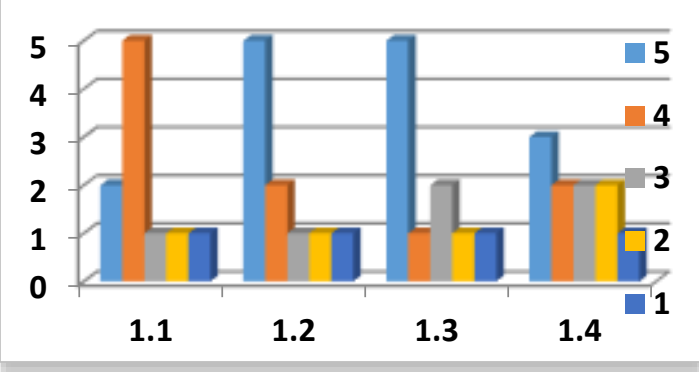

Figure 2. Graph of Training Program

\section{Process Standard}

\section{Development of Training Quality}

The evaluation results show the answers of items (2.1) clarity and completeness of policy documents regarding the establishment and existence and function of the field of quality control exercise that encourages athletes to work hard, independent, working, experimenting with utilizing various resources that results are used by the coach and the Organization, $(50 \%)$ respondents chose item no (4). The answers of item test (2.2) the clarity of quality of sport training system implemented by organization including monitoring process, evaluation, and its utilization, (60\%) respondents chose item no (5). The answer of item test (2.3) presentation of implementation of training method which used sciences approach from all over the sports, (70\%) respondents chose item no $(50 \%)$. The answers of item test (2.4) the amount of involvement of experts as keynote speakers for improving the quality of training, (40\%) respondents chose item no (5). The result of tabulation can be seen down below:

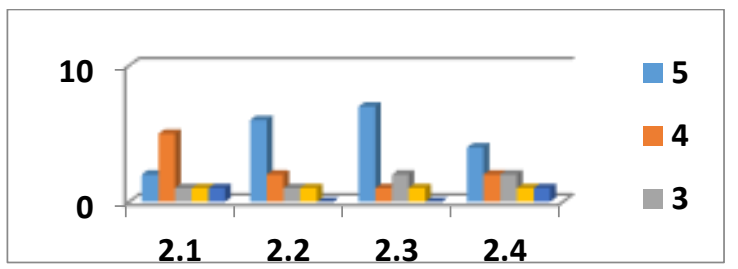

Figure 3. Graph of the development of training quality

\section{Result Standard}

\section{Athletes Target Orientation}

The evaluation results show the answers of items (3.1) Percentage of athletes who failed to achieve the targets set in all sports, $(40 \%)$ of respondents chose no lattice (5). Answer of items (3.2) Percentage of athletes who managed to achieve the targets set in all sports, $(40 \%)$ of respondents chose no lattice (4). The tabulated results can be seen as follows:

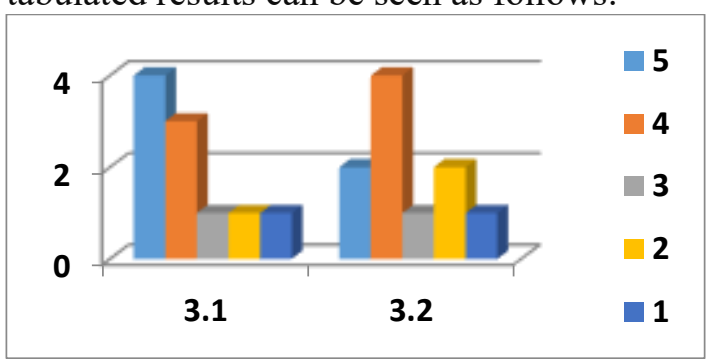

Figure 4. Graph of Athletes Target Orientation

\section{Athletes Achievement}

The evaluation results show the answers of items (4.1) Achievement of the athlete's performance in the provinces / territories, 
(70\%) of respondents chose no lattice (5). Answer of items (4.2) Achievement of the athlete's performance at the national level $(60 \%)$ of respondents chose no lattice (5). Answer of items (4.3) Achievement of the athlete's performance at the international level $(50 \%)$ of respondents chose grilles no (2).Answer of items (4.4) Organizational Efforts to improve the acceleration of the athlete's performance, $(60 \%)$ of respondents chose no lattice (5) The tabulated results can be seen as follows:

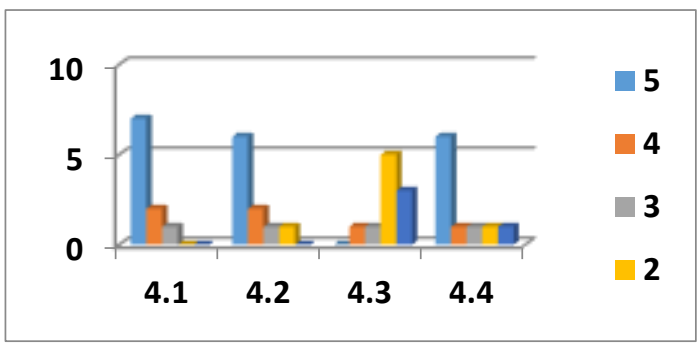

Figure 5. Graph of Athletes Achievement

\section{Human Resources Standard The availability of coaches and supporters.}

The evaluation results show the answers of items (5.1) The availability of trainers who meet the qualifications, $(50 \%)$ of respondents chose no lattice (4). Answer of items (5.2) Availability of support personnel such as (1) masseure, (2) physical trainer, (3) medical, (4) experts and (5) psychologists in all sports, $(60 \%)$ of respondents chose grating no (5). Answer of items (5.3). Availability of management system trainers and support personnel, $(70 \%)$ of respondents chose no (5). Answer of items (5.4) Availability of guidelines and documents concerning the implementation of monitoring and evaluation systems, as well as the track record of the performance of coaches and support personnel, $(40 \%)$ of respondents chose no (5). The tabulated results can be seen as follows:

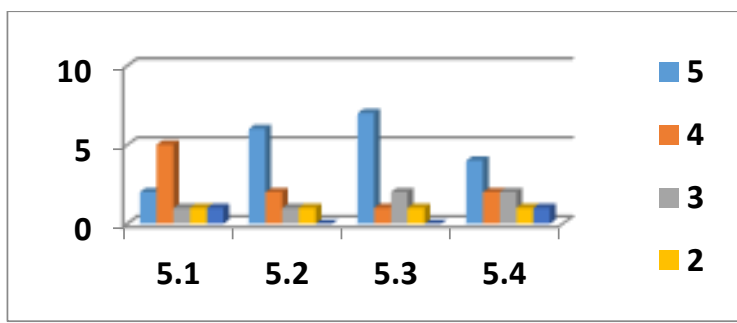

Figure 6. Graph of the availability of coaches and support personel

\section{Facilities And Infrastructures Standard}

The availability of facilities and infrastructures

The evaluation results show the answers of items (6.1) The availability of trainers who meet the qualifications, $(50 \%)$ of respondents chose no lattice (4). Answer of items (6.2) Availability of support personnel such as (1) maseur, (2) physical trainer, (3) medical, (4) experts and (5) psychologists in all sports, $(60 \%)$ of respondents chose grating no (5). The tabulated results can be seen as follows:

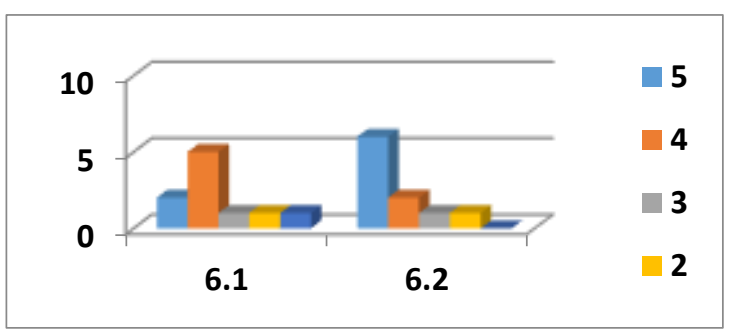

Figure 7. Graph of the availability of facilities and infrastructures

\section{Management Standard Governance Structure}

The evaluation results show the answers of items (7.1) Completeness and clarity of organizational system that allows the implementation consistently, (50\%) of respondents chose no (4). Answer of items (7.2) Clarity organization's management system, $(60 \%)$ of respondents chose no (5). The tabulated results can be seen as follows:

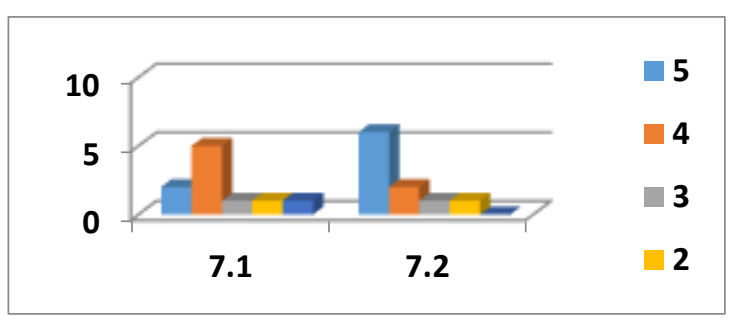

Figure 8. Graph of Governance Structure

\section{Management System}

The evaluation results show the answers of items (8.1) Clarity of job analysis, job descriptions, program improvement of managerial competence, $(50 \%)$ of respondents chose no (4). The tabulated results can be seen as follows: 


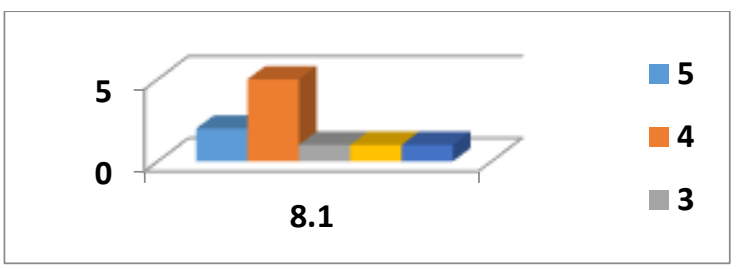

Figure 9. Graph of Management System

\section{Athletes Selection}

The evaluation results show the answers of items (9.1) Selection System athletes who apply the principles of equity, (50\%) of respondents chose no lattice (4). Answer of items (9.2) Governance new athletes reception system that includes policies, criteria, procedures, instruments, systems and consistency of implementation decisions, $(60 \%)$ of respondents chose no lattice (5). Answer of items (9.3) System of information, $(70 \%)$ of respondents chose no (5). Answer of items (9.4) The selection, (40\%) of respondents chose no (5). Answer of items (9.5) Availability of services to athletes in the field of mental guidance, Health / insurance, rewards, and guaranteed employment, (40\%) of respondents chose no (5). The tabulated results can be seen as follows:

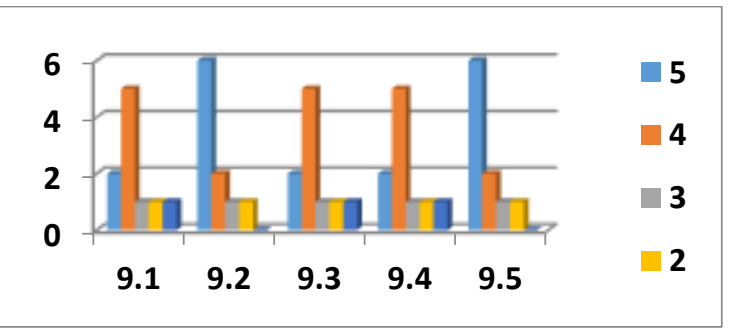

Figure 10. Graph of Athletes Selection

\section{Quality Assurance System}

The evaluation results show the answers of items (10.1) The existence of internal quality assurance system complete with criteria and assessment instruments as well as its use to measure the performance of each unit, as well as dissemination of the results, $(50 \%)$ of respondents chose no (4). Answer of items (10.2) The existence of internal quality evaluation system complete with criteria and assessment instruments as well as its use for measuring the performance of the organization, $(60 \%)$ of respondents chose no (5). The tabulated results can be seen as follows:

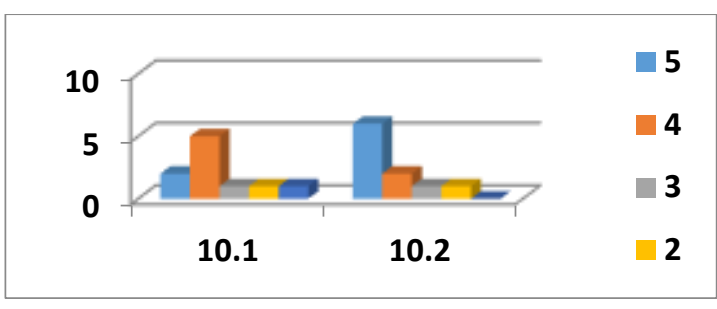

Figure 11. Graph of Quality Assurance System

\section{Financing Standard Fund Management}

The evaluation results show the answers of items (11.1) Clarity and completeness of fund management policy documents, $(50 \%)$ of respondents chose no (4). Answer of items (11.2) Percentage of resources obtained from the cost of the grant, the result of cooperation sponsors, communities and so per year, $(60 \%)$ of respondents chose no (5). Answer of items (11.3)

Clarity of guidelines for financial accountability in accordance with applicable regulations, $(70 \%)$ of respondents chose no (5). Answer of items (11.4) setting mechanisms athlete training costs, $(60 \%)$ of respondents chose no (5). Answer of items (11.5) Clarity of policy and financing mechanisms athlete, $(60 \%)$ of respondents chose no (5). Answer of items (11.6) Clarity system of monitoring and evaluation of internal funding, $(50 \%)$ of respondents chose no lattice (5). Answer of items (11.7) The financial statements are transparent and accessible to all stakeholders (40\%) of respondents chose no lattice (5). The tabulated results can be seen as follows:

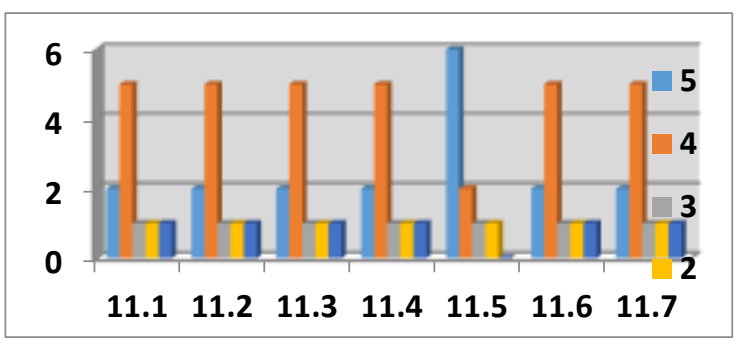

Figure 12. Graph of Fund Management

\section{Evaluation Standard Evaluation of Exercises Result}

The evaluation results show the answers of items (12.1) Conformity evaluation system training results with the target achievement of physical, technical and mental set, $(50 \%)$ of respondents chose no lattice (4). Answer of items (12.2) Conformity evaluation system 
training results with appropriate measurement model, $(60 \%)$ of respondents chose no lattice (5). The results can be seen in the following tabulation.

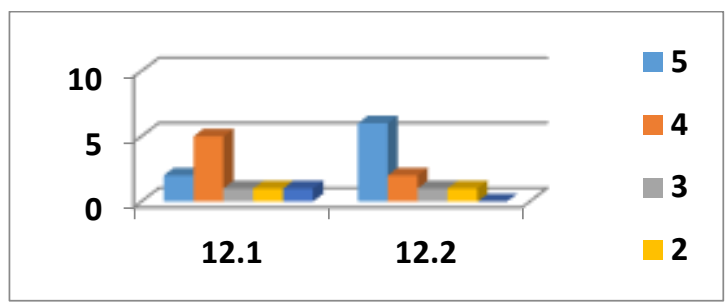

Figure 13. Graph of Evaluation of Exercises Result

\section{Cooperation Standard} Cooperation Achievement

The evaluation results show the answers of items (13.1) policy, management, and monitoring and evaluation by the Organization in cooperation activities, (50\%) of respondents chose no (4). Answer of items (13.2) Number of cooperation in the last three years $(60 \%)$ of respondents chose no (5). Answer of items (13.3) Percentage of coaches who are members of sports organizations or other professional sports field during the last three years $(70 \%)$ of respondents chose no (5). Answer of items (13.4) Number of international level athletes out of total athletes (40\%) of respondents chose no (5). The tabulated results can be seen as follows:

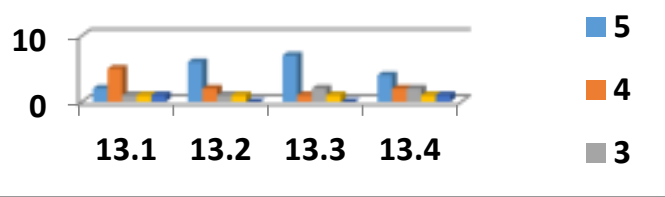

Figure 14. Graph of Cooperation Achievement

\section{Discussion}

The results showed that, peoples need towards achievements in sports are rather significant Ghotbabadi (2012). This is in accordance withthe results of Team Economica Paper's Research and "Badan Otonom Economica Indonesia" of Economic Faculty, University of Indonesia in 2011, that conlude as follows: $59 \%$ improved, $24 \%$ the same and $17 \%$ worsened. While based satisfaction index of Indonesian society towards sports Indonesia with variable Achievement (71.85\%), Event (79.24\%), Impressions $(77.13 \%)$, Facilities (59.36), Development (63.86\%), welfare $(58.73 \%)$ and regeneration $(67.09 \%)$.
Model quality evaluation exercise show that needs to be done throughout the sports stakeholders in an effort to strengthen the system of tiered and sustainable development powel (2015), Khatmi (2012). It is appropriate Law Number 3 of 2005 states that sporting achievement is a sport that foster and develop the sportsman in a planned, tiered and sustainable.

In order to ensure enforceability and optimum results in the field of sporting achievement necessary integrated quality control system throughout the sport's governing achievement. The results showed that exercise training quality evaluation model of achievement as follows; (1) Formulation of quality document consists of; (a) a quality policy, (b) a quality manual, (c) quality standards, and (d) quality control instruments. (2) internal quality assurance system, (3) an external quality assurance system. These three aspects into one inseparable unity with the aim of gradually improving quality and sustainable. This is consistent with the concept of internal quality assurance system of higher education (2010).

The results showed that the percentage of achievement of management of sport organizations is generally low. This condition is caused not yet integrated quality assurance system that can guarantee the quality of each variable management organization of sporting achievement.

\section{Conclusion}

First, the survey results of sports level of understanding the organization's management achievements in general do not understand what, how, why and their importance continuous quality assurance. This needs to be given an understanding for the management of the importance of quality in the process of exercise accomplishment. Needs of the importance of quality evaluation found that in general say the quality evaluation of exercise is needed. It is important to apply a quality evaluation model to evaluate the quality of exercise training.

Second, sports coaching achievements made in North Sumatera already exist although not evenly across all districts of the city, but would be very good if the quality of coaching is controlled and improved continuously.

Third, the approach to evaluation carried 
out so far is merely incidental and follow-up as well not exist.

Fourth, quality evaluation models need to be designed workouts which oriented continuously to quality improvement by implementing good practices through internalization of culture to quality and recognition from outsiders.

\section{References}

Abdullah Dhukail at Khatmi (2012) Evaluation of Studi family medicine training program: The aplication of CIPP evaluation formal, Journal Medical Teacher, Vol. 34 Issue Sup 1.

Alfini A., Yuliandra E., Desi S. (2012). Olahraga Prestasi Indonesia. Badan Otonom Economica, FE Universitas Indonesia

(scribd.com/dokumen/104942171/Ola hraga-Indonesia-Tulkisan-Utama-EP54.

Azmi, Chairul and Sunarno, A (2017). Intensive Training Program Evaluation of the Indonesian National Sport Commitee of North Sumatera, International Journal of Science and Reseach, ISSI (online) 2319-7064,
Vol. 6 Issue 4 April 2017

Borg W.R and Gall M.D (1983). Educational Research: An Introduction. New York: London. Longman.

Brady, M. K., \& Cronin, J. J. (2001). Some new thoughts on conceptualizing perceived service quality: Ahierarchical approach. Journal of Marketing, 65(3), 34-49.

Fernandes H.J.X (1986), Evaluation of Educational Program: Jakarta: National Educational Planinng, Evaluation and Curiculum Development.

Ghotbabadi A.R., Baharun R and Feiz (2012), Areview of Service Quality Models, Proceeding $2^{\text {nd }} \quad$ International Conference on Management ( $2^{\text {nd }}$ ICM 2012),

www.internationalconference.com.my. Langkawi Kedah, Malaysia.

Powel, Brent (2015) Utilizing the CIPP Model as a Means to Develop and Integrated Service-Learning Component in a Univercity Health Course, Journal of Health Education Teaching, Vol 6 (1): 21-3 